\title{
Analysis of Accurate Learning in Radial Basis Function Neural Network Using Cosine Similarity on Leaf Recognition
}

\author{
Amir Saleh ${ }^{1}$, Tulus ${ }^{1}$, and Syahril Efendi ${ }^{1}$ \\ \{amirsalehnst1990@gmail.com \} \\ ${ }^{1}$ Department of Computer Science and Information Technology, Universitas Sumatera Utara, Medan, \\ Indonesia
}

\begin{abstract}
RBF network is a method of artificial neural networks that perform hybrid learning, namely supervised learning and unsupervised learning. Basically, problem found in learning of RBF network that is a difficult to determine the centroid exactly and weight of network the less optimal. Generally, to determine the centroid is done by random and updated to use k-means clustering method. However, selection of initial centroid is less precise will have an effect on the decrease of accurate learning on leaf recognition in RBF network. A method is used to measure the level of similarity between the data proposed in this research, ie cosine similarity. By comparing the distance of similarity between each data, will be generated data the highest level of similarity from the others, so that can be used as a centroid in RBF networks. The result of research RBF network using cosine similarity will be compared a common method used in centroid determination, ie k-means clustering. The results of the test on data showed that leaf recognition with RBF network using cosine similarity method obtained an accuracy level of $79.22 \%$, while the leaf recognition with RBF network using k-means clustering method obtained an accuracy level of $63.91 \%$.
\end{abstract}

Keywords: RBF network, centroid, k-means clustering, leaf recognition, cosine similarity

\section{Introduction}

Neural network is one method of artificial intelligence that is used in pattern recognition by imitating the work of the human nervous system. Humans can recognize between one object and another by storing knowledge that is experience and reused in the time required [1]. Meanwhile, artificial neural networks that are operated on the machine to recognize an object is done by recognizing the pattern of the object and train the network to obtain the weights that are used again in the process of testing the pattern recognition of the next object. By comparing the pattern with one another, then obtained a similar pattern that can be insert into a classification.

A method is used in neural networks which has certain advantages over other types of neural networks including better approximation capabilities, simple network structure, and faster learning is radial basis function (RBF) neural network [2]. This method performs a hybrid learning, which is a supervised and unsupervised learning. 
In the learning process, RBF network requires centroid. One of the common problems found in this network is the difficulty of determining the centroid exactly. The common method is used in determining centroid is k-means clustering. The determination of the begining value of the initial centroid greatly affects the results [3]. The method of initial centroid determination is commonly done by random. The next process is to update the centroid value so that the final centroid value is better. However, when determining the initial centroid is less precise will give the result of pattern recognition accuracy is not optimal. These centroid should be placed in a cunning way because of different location causes different result [2]. From the results of experiments have been conducted by taking some samples of centroid randomly, the result of accuracy of pattern recognition is different. This is proven to determine the centroid can affect the performance of RBF network.

Some previous researchers have done the research to determine the centroid inthe RBF network. One of them is the determine of centroid done with Particle Swarm Optimization (PSO). The result testing has done using centroid with PSO can obtain better results than commonly used centroid in the RBF network[3]. Other research that using fuzzy in determining centroid in the RBF network. The results are better performance of RBF network than using the usual method in determination of centroid [5].

In this research will be proposed an algorithm used to measure the level of similarity of data, ie cosine similarity in determination of centroid in RBF network. It will be used in the classification data and determined the exact centroid location. Prior to measuring the level of similarity of data, the leaf of image are introduced its pattern, so that the data in the form of numeric can be processed.

The leaves of images to be recognized pattern must be extracted features with some image processing methods. An image will be characterized by its shape, texture and color. Shape features can be identified from the value of roundness and eccentricity in an image [6]. Texture features to use GLCM method which will be recognized from the value of contrast, correlation, energy, and homogeneity [4]. Meanwhile, the color features is obtained by using HSV method which convert RGB image to HVS which will be recognized from the value of hue, saturation, and value [5].

Similar to Euclidean distance, cosine similarity is also used to calculate the distance between each data. Higher values will show a higher level of similarity. The use of cosine similarity in this study did not require the initial centroid. It because the data used is already classified, the next find the exact data to be used as a centroid. Centroid will be generated by comparing the similarity between each data in one classification. Data that has a higher similarity than the other data that will be selected to be centroid. This process is done as many as the data classification used in RBF network training. The collection centroid from each classification has function as a centroid in the learning of RBF network to know the accuracy of leaf recognition. Each centroid will has the collection data that has a similarity to it to be grouped in the classification. The use of cosine similarity method is chosen as the method of measuring the similarity of data due to better accuracy in each testing than the Euclidean distance method in the measurement of similarity level [6].

\section{Radial basis Function}

The RBF network is a kind of three-layer forward neural network, the hidden layer activation function of which is a set of radial transmitted to the hidden cell space. This set of kernel function forms a base of the input sample (Pan, et al., 2011). Architecture of RBF can be seen in Figure 1 as follows(Li, et al., 2013): 


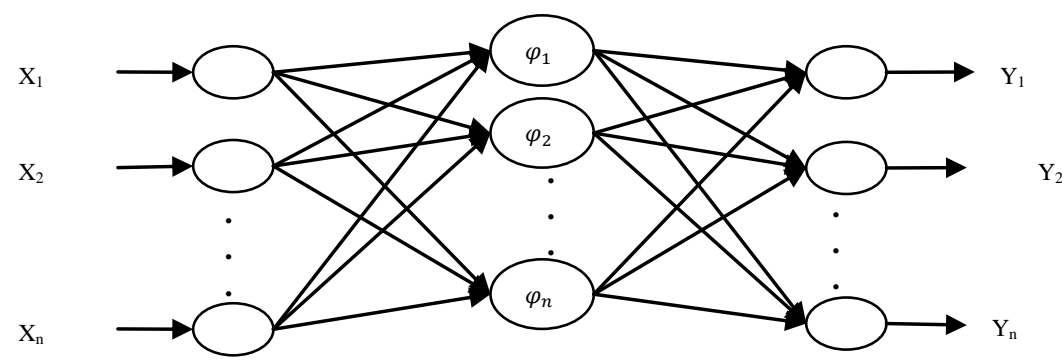

Figure1. Architecture of RBF Network

\subsection{Input layer}

Input layer is part in RBF network input in the first layer. The other side, input in RBF networkis used as input data that is function to determine centroid and the standard deviation from data that generate activation function of hidden layer. There are two ways that is used in determining centroid, ie random and k-means clustering methods.

The random method is performed by selecting data in the vector input randomly. This method is easy to implement but less precise in the selection of centroid in input data. The second, using k-means clustering method, that is grouping the data according into its cluster and determining the data itself which will be made its centroid. This method is difficult to implement but will result the exact centroid. Looking at the first step of the RBF network learning, it can be concluded that at the step of the training is unsupervised. The acquired centroid will be used for the activation function in hidden layer. The selection of the exact centroid will result the good calculation and the appropriate weight gain so that the pattern can be well recognized in the RBF network.

After searching the centroid value of data input using k-means clustering algorithm, the next step is calculated Gaussian value by using Gaussian equation. The centroid value of the search results from the input data is used in hidden layer. Before calculating the Gaussian value, the standard deviation value is determined first.

\subsection{Hidden layer}

The centroid value is obtained from the input layer will be processed on hidden layer in RBF network. In this layer the activation function is used to obtain the network weights used in the output layer and formulation activity in the formation of the algorithmic system used in the RBF network. Commonly, implementation is used functions in solving the case using RBF network is by using Gaussian basis function which is formulated as follows:

where,

$$
\varphi(r)=\exp \left(-\frac{\left\|x_{i}-c_{i}\right\|^{2}}{2 \sigma_{i}^{2}}\right)
$$

$\mathrm{X}=$ Input data

$\mathrm{c}=$ Centroid

$\sigma=$ Spread

with, 


$$
=\frac{d_{\max }}{\sqrt{n}} \quad \sigma=\frac{\text { Maximum distances of } 2 \text { center }}{\sqrt{\text { ncenter }}}
$$

After the Gaussian value is known, the next process, calculate the new weight (w) by multiplying the pseudoinverse of the $G$ (Gaussian) matrix by the target vector (d) by the following equation:

$$
w=\left(G^{T} G\right)^{-1} G^{T} d
$$

After the weight value is known then the next step is to store the value of centroid calculation results and weights value to be reused during the testing process in RBF network.

\subsection{Output Layer}

The last step, after obtain the result calculation of Gaussian's basis function by weights updating. The sum of the multiplication of weights with the basis function will produce an output called the output layer. The output layer responds from the network according to the pattern previously entered on the input layer. The transformation from the input layer into the hidden layer is non-linear, while the transformation from the hidden layer into the output layer is linear. This step, it is done to calculate the network output value of $y(n)$ is summed the bias weights (b) as follow as:

$$
y(n)=\sum_{i=1} w G(\|x-c\|+b)
$$

\section{Cosine Similarity}

Each object of one another can be grouped in the same type based on the similarity of the pattern formed. The object is translated by image processing method. The image is processed using the technique can be identified with each other based on shape, texture, and colorthat contained in an image. While techniques to determine the similarity or image similarity so that can be grouped in one type can use a method of similarity, one of which is cosine similarity.Cosine similarityis a method that used to measure a level of similarity between two data [9]. The purpose of this method compares two objects to be calculated the level of similarity, so that can be seen how the level of similarity. In some cases, it is also often applied to calculate the level of similarity of documents or images. It is similar to the Euclidean distance method is used to measure the distance between two pieces of data.The difference is that the process of measuring the distance in the Euclidean distance method is used the minimum distance to measure the level of similarity level, while the cosine similarity method is used the maximum distance. Mathematically to calculate the level of similarity is as follows:

$$
\operatorname{Similarity}(X, Y)=\frac{\sum x_{i} y_{i}}{\sqrt{\sum x_{i}^{2}} \cdot \sqrt{\sum y_{i}^{2}}}
$$


Equation of cosine is called have a data similar, if the angle is 0 degrees and the similarity is 1 and when two data are not similar at all, the angle is 90 degrees and the similarity is 0 .

\section{Pattern Recognition}

Artificial neural network has been used widely in pattern recognitionandcommonly shows advantages compared with other learning methods, general nature, and adaptabilityin mapping non-linearly [1]. Pattern recognition is a subject researching object description and classification method, it is also a collection of mathematical, statistical, heuristic, and inductive techniques of fundamental role in executing the tasks like human being on computers [3].

Before insert into the artificial neural network, the first it must introduce the image pattern. In this paper is used to features an image based on the shape, texture, and color in the image. Figure 2 shows the block diagram used for image processing.

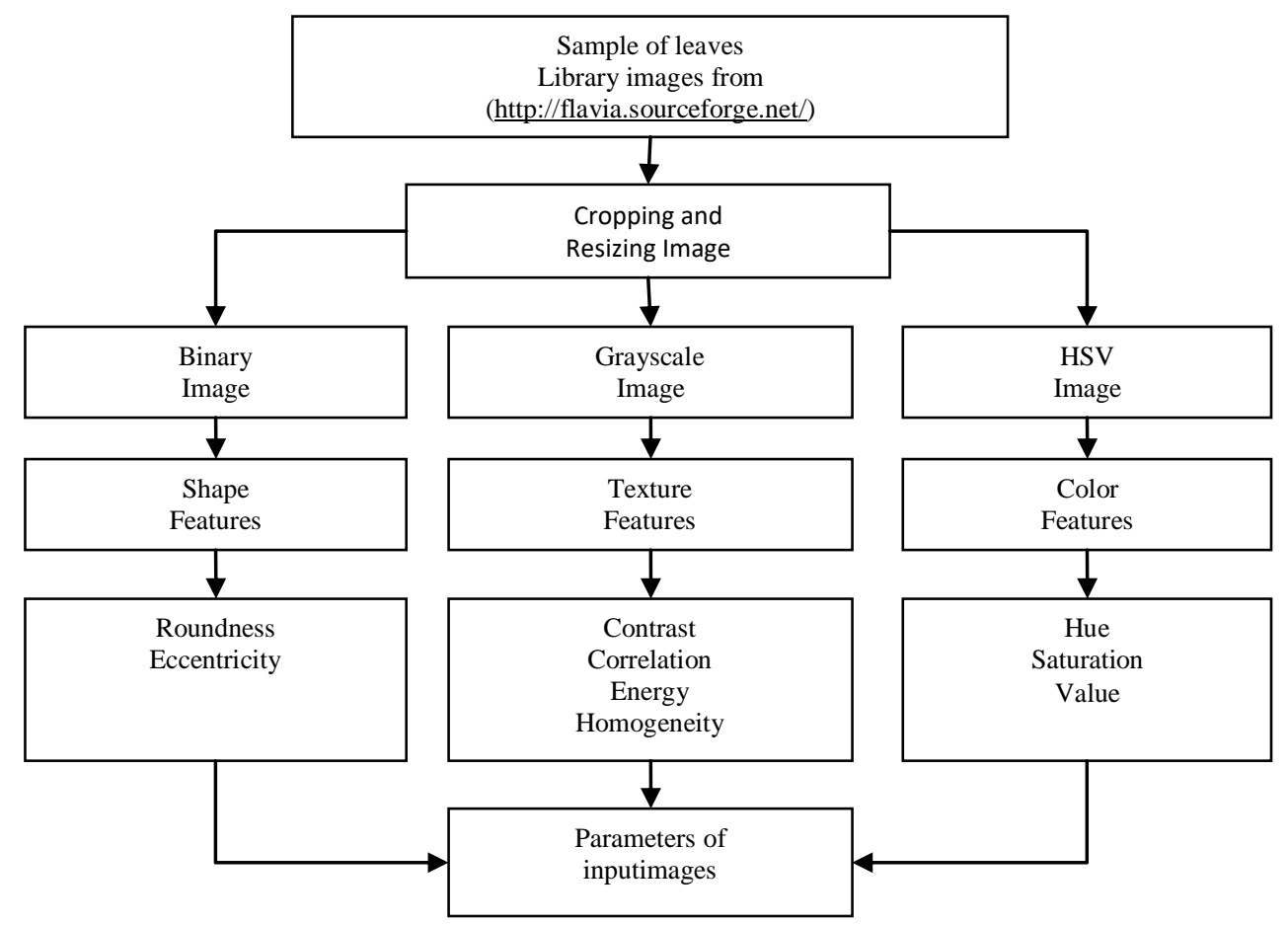

Figure 2. Digital image processing block diagram

The leaves of images obtained on Flavia dataset [10]. All leaves of images are in 512 x 512 resolution. An RGB image is firstly converted into grayscale, binary, and HSV image to obtained features value. Figure 3 shows the result of convertion on the RGB image. 


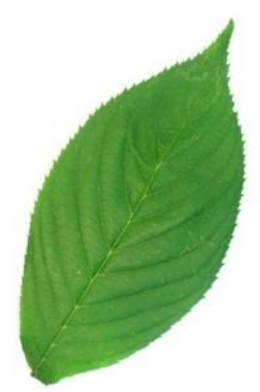

Figure 3. RGB image

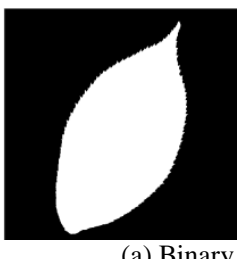

(a) Binary

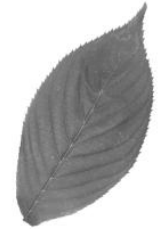

(b) Grayscale

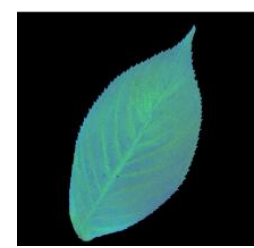

(c) HSV

Figure 4:Image conversionondigital image processing

\subsection{Shape Features}

Shape features extraction is one way used to extract the image features associated with the geometric of the image (Kadir, et al., 2011). Type of leaf feature extraction is used consists of several features, as follows(Kumar, et al., 2015):

$$
\begin{gathered}
\text { Roundness }(\gamma)=\frac{A}{P}=\frac{4 \pi x A}{P^{2}} \\
\text { Eccentricity }(e)=\sqrt{1-\frac{\text { Major Axis Length }}{\text { Minor Axis Length }}}
\end{gathered}
$$

From the both of it will produce two input images $\left(\mathrm{x}_{1}\right.$ and $\left.\mathrm{x}_{2}\right)$ which will be processed into the RBF network. Every image will be processed into the RBF network will be generated the both of it.

\subsection{Texture Features}

The next step is to extract the image to obtain texture featuresof the image. Generally, the method is used the Gray Level Cooccurrence Matrix (GLCM). GLCM is presented by Haralick [5], is one of the statistical methods for textural feature extraction and includes the most widely method is used. The GLCM method in this researchusing various features of texture which is representation of digital image which want to be analyzed. The features of these textures, as follows[4]: 


$$
\begin{aligned}
& \text { Contrast }=\sum_{i} \sum_{j}(i-j)^{2} P(i, j) \\
& \text { Correlation }=\frac{\sum_{i} \sum_{j} i, j P[i, j]-\mu_{i} \mu_{j}}{\sigma_{i} \sigma_{j}} \\
& \text { Energy }=\sum_{i} \sum_{j} P[i, j]^{2} \\
& \text { Homogeneity }=\sum_{i} \sum_{j} \frac{P[i, j]}{1+[i-j]}
\end{aligned}
$$

The GLCM method is used in data input will produce 4 texture extraction. Each image will be processed has a value based on contrast, correlation, energy, and homogeneity is contained in the images. The feature is used as input in RBF network with $\mathrm{x}_{3}, \mathrm{x}_{4}, \mathrm{x}_{5}$, and $\mathrm{x}_{6}$ symbols respectively.

\subsection{Color Features}

The color features extraction in images is done in this research by looking for way of extraction of the color characteristicswith the HSVmethod. Where this method is used to define the RGB color that becomes the value of Hue, Saturation, and Value. The RGB value contained in an images has a range between $0-255$ to be converted to HSV with a value of 01. The formulas used are[5]:

$$
\begin{aligned}
& R^{\prime}=\frac{R}{255} ; G^{\prime}=\frac{G}{255} ; B^{\prime}=\frac{B}{255} \\
& C \text { max }=\max \left(R^{\prime}, G^{\prime}, B^{\prime}\right) \\
& \text { Cmin }=\min \left(R^{\prime}, G^{\prime}, B^{\prime}\right) \\
& \Delta=C \max -\text { Cmin }
\end{aligned}
$$$$
\left\{\begin{array}{c}
60^{\circ} \times\left(\frac{G^{\prime}-B^{\prime}}{\Delta} \bmod 6\right), \text { Cmax }=R^{\prime} \\
\mathrm{H}(\text { Hue })=60^{\circ} \times\left(\frac{B^{\prime}-R^{\prime}}{\Delta}+2\right), \text { Cmax }=G^{\prime} \\
60^{\circ} \times\left(\frac{R^{\prime}-G^{\prime}}{\Delta}+4\right), C \max =B^{\prime}
\end{array}\right.
$$$$
\frac{\Delta}{C \max }, \Delta<>0 \quad\left\{\begin{array}{c}
0 \quad, \Delta=0 \\
S(\text { Saturation })=
\end{array}\right.
$$

$$
V(\text { Value })=\text { Cmax }
$$

From the calculation of this HVS method, will produce color features are written with the symbols $\mathrm{x}_{7}, \mathrm{x}_{8}$, and $\mathrm{x}_{9}$. Each input images will produce color features before being processed in the RBF network. 


\section{Proposed Approach}

To analyze the accuracy learning in RBF network using cosine similarity method in centroid determination on leaf recognition, the data is used in the form leaves of images is obtained from internet page with address: http://flavia.source-forge.net/, with the selection and cutting of images size of 512 pixels x 512 pixels, which will be formed into shape, texture, and color extraction matrix.

The amount of data is used as many as 32 types leaves of images and each species will be taken as many as 50 leaves of images, so the total data are 1600 images. The image processing data is done by taking the extraction value of the shape, texture, and colorleaves of images which will be divided into two parts, are data training and testing. The division of data is 60 : 40, where data training is used as many as 960 leaves of images and data testing is used as many as 640 leaves of images. The proposed approch can be seen in Figure 5 as follows:

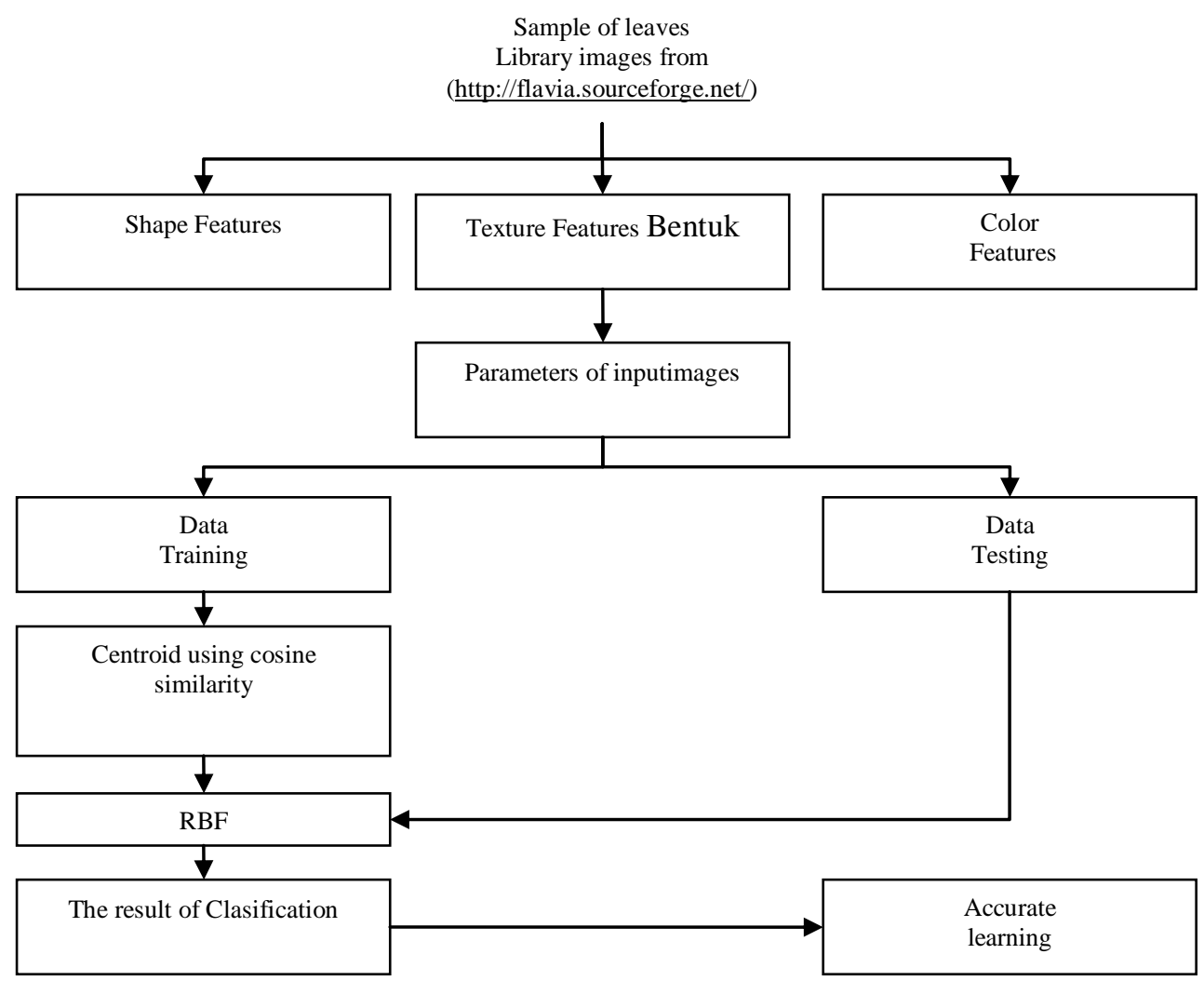

Figure 5. The proposed metodology

Figure 5 illustrates the process of input images matrix recognition is done by taking the features extraction value of each image.The features extraction is used of the shape, texture, and color of the 9 pieces of value or parameters in each image. The next process is the centroid determination that will be done by using the general method, is k-means clustering 
and cosine similarity method to obtain the good centroid is based on data similarity. All of the calculation process will produce weights vector that will be inserted into RBF recognition for the classification step. This step will process the pattern recognition of input and output, to compare with the target so that it can be calculated the accuracy of learning. Mathematically equation is used to calculate the results of leaf recognition can be formulated as follows:

$$
\text { Accuracy }(\%)=\frac{\text { The numbers of true data }}{\text { All numbers of data }}
$$

\section{Experiment Result}

In the process of leaf recognition using $\mathrm{RBF}$ network in this research, the centroid determination is done by using cosine simalrity method and comparing it with k-means clustering method. The results of leaf recognition using RBF network with cosine similarity and $\mathrm{k}$-means clustering can be seen in table 1 .

Table 1. RBF network testing

\begin{tabular}{|c|l|c|c|}
\hline No. & \multicolumn{1}{|c|}{ Method } & Recognition & Accuracy of network testing \\
\hline 1. & RBF-KMeans & 409 & $63.91 \%$ \\
\hline 2. & RBF-Cosine & 507 & $79.22 \%$ \\
\hline
\end{tabular}

The based of analysis results of accuracy learning using usual of RBF network and RBF network using cosine similarity have different results. Using k-means clustering in the RBF network has an accuracy of $63.91 \%$. It is caused centroid calculation results have not achieved optimal results. The based of several experiments are conducted, to obtain the initial centroid with different data on k-means clustering calculation results will result in a different amount of accuracy. It needs to be thoroughly tested on all input data and determine which data is appropriate to be the initial centroid. This process will result in a long time due to the calculation and comparison process in centroid determination.

While the results of cosine similarity in RBF network obtained an accuracy of $79.22 \%$. This is because the centroid selection is only done on similar data that has been visually classified. The use of this method only compares the input data in a class to compare the level of similarity with other data. So the selected centroid is just a data in the same class.

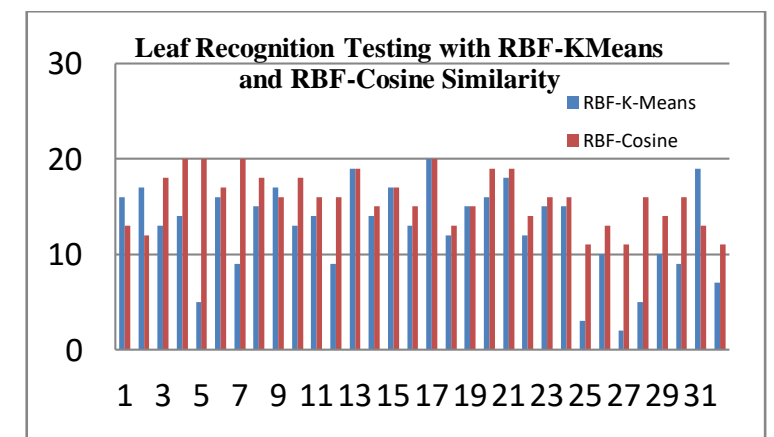

Figure 6. Leaf recognition testing 


\section{Conclusion}

The based of the results are obtained by comparing k-means clustering and cosine similarity method in centroid determination in RBF network, the increase using cosine similarity method is higher by $15.31 \%$ if it is compared to the usual RBF network. It proves that the leaf recognition requires the good centroid to improve the performance in $\mathrm{RBF}$ network.

\section{References}

[1] S. Haykin, "Neural networks-A comprehensive foundation," New York: IEEE Press. Herrmann, M., Bauer, H.-U., \& Der, R, vol. psychology. p. pp107-116, 1994.

[2] S. P. Munnoli and a U. Bapat, "Clustering Algorithms for Radial Basis Function Neural Network," ITSI Trans. Electr. Electron. Eng., pp. 113-116, 2013.

[3] M. Foqaha and M. Awad, "Hybrid Approach to Optimize the Centers of Radial Basis Function Neural Network Using Particle Swarm Optimization,” vol. 12, no. 5, pp. 396-407, 2017.

[4] M. Zulfaezal, C. Azemin, M. Izzuddin, M. Tamrin, M. R. Hilmi, and K. M. Kamal, "glcm texture analysis on different color space for pterygium grading," vol. 10, no. 15, pp. 6410-6413, 2015.

[5] M. Deswal and N. Sharma, "A Fast HSV Image Color and Texture Detection and Image Conversion Algorithm,” Int. J. Sci. Res. ISSN (Online Impact Factor, vol. 3, no. 6, pp. 23197064, 2012.

[6] K. Zuva and T. Zuva, "Effectiveness of Image ( dis ) similarity Algorithms on Content- Based Image Retrieval," Int. J. Eng. Sci., vol. 1, no. 1, pp. 31-35, 2012.

[7] Y. Pan, W. Xue, Q. Zhang, and L. Zhao, "A forecasting model of RBF neural network based on genetic algorithms optimization," 2011 Seventh Int. Conf. Nat. Comput., pp. 48-51, 2011.

[8] B. Li, L. Cong, and W. Zhang, "Research on Optimized RBF Neural Network Based on GA for Sewage Treatment," 2013 5th Int. Conf. Intell. Human-Machine Syst. Cybern., pp. 520-523, 2013.

[9] S. Kaur and D. Aggarwal, "Image Content Based Retrieval System using Cosine Similarity for Skin Disease Images," Adv. Comput. Sci. an Int. ..., vol. 2, no. 4, pp. 89-95, 2013.

[10] S. G. Wu, F. S. Bao, E. Y. Xu, Y. Wang, Y. Chang, and Q. Xiang, "A Leaf Recognition Algorithm for Plant Classification Using Probabilistic Neural Network,” Int. Symp. Signal Process. Inf. Technol., pp. 11-16, 2007.

[11] A. Kadir, L. E. Nugroho, A. Susanto, and P. I. Santosa, "Leaf Classification Using Shape, Color, and Texture Features," Int. J. Comput. Trends Technol., no. July to Aug, pp. 225-230, 2011.

[12] R. Kumar, R. Srivastava, and S. Srivastava, "Detection and Classification of Cancer from Microscopic Biopsy Images Using Clinically Significant and Biologically Interpretable Features," J. Med. Eng., vol. 2015, pp. 1-14, 2015.

[13] R. Haralick, K. Shanmugan, and I. Dinstein, "Textural features for image classification," IEEE Transactions on Systems, Man and Cybernetics, vol. 3. pp. 610-621, 1973. 